\title{
Erratum to: e-GRASP: an integrated evolutionary and GRASP resource for exploring disease associations
}

Sajjad Karim¹, Hend Fakhri Nour Eldin', Heba Abusamra', Nada Salem¹, Elham Alhathli', Joel Dudley², Max Sanderford ${ }^{3}$, Laura B. Scheinfeldt ${ }^{3,4}$, Adeel G. Chaudhary' ${ }^{1}$, Mohammed H. Al-Qahtani ${ }^{1}$ and Sudhir Kumar ${ }^{1,3,4^{*}}$

\section{Erratum}

This article [1] unfortunately published with an author deleted in the author list. The correct author list is presented above.

The author list was previously incorrectly presented as:

Sajjad Karim ${ }^{1}$, Hend Fakhri NourEldin ${ }^{1}$, Heba Abusamra ${ }^{1}$, Nada Salem ${ }^{1}$, Elham Alhathli ${ }^{1}$, Joel Dudley ${ }^{2}$, Max Sanderford ${ }^{3}$, Laura B. Scheinfeldt ${ }^{3,4}$ and Sudhir Kumar ${ }^{1,3,4^{*}}$

${ }^{1}$ Center for Excellence in Genome Medicine and Research, King Abdulaziz University, Jeddah, Saudi Arabia. ${ }^{2}$ Department of Genetics and Genomic Sciences, Mount Sinai School of Medicine, New York, NY 10029, USA. ${ }^{3}$ Institute for Genomics and Evolutionary Medicine, Temple University, Philadelphia, PA 19122, USA. ${ }^{4}$ Department of Biology, Temple University, Philadelphia, PA 19122, USA.

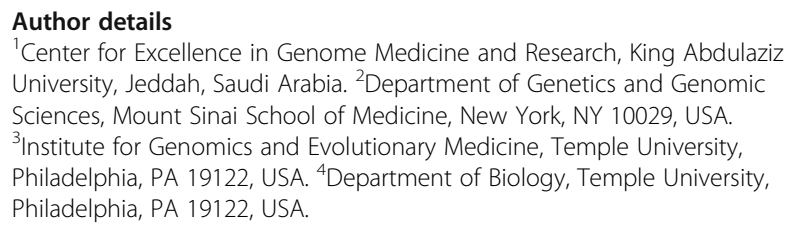

Received: 8 March 2017 Accepted: 8 March 2017

Published online: 06 April 2017

\section{Reference}

1. Karim S, Nour Eldin HF, Abusamra H, Salem N, Alhathli E, Dudley J,

Sanderford M, Scheinfeldt LB, Chaudhary AG, Al-Qahtani MH, Kumar S.

e-GRASP: an integrated evolutionary and GRASP resource for exploring

disease associations. BMC Genomics. 2016;17 Suppl 9:770.

\footnotetext{
* Correspondence: s.kumar@temple.edu

${ }^{1}$ Center for Excellence in Genome Medicine and Research, King Abdulaziz University, Jeddah, Saudi Arabia

${ }^{3}$ Institute for Genomics and Evolutionary Medicine, Temple University,

Philadelphia, PA 19122, USA
} 